\title{
Geometric model for the critical-value problem of nucleation phenomena containing the size effect of nucleating agent
}

\author{
Donghua $\mathrm{Xu}^{*}$ and William L. Johnson \\ Division of Engineering and Applied Science, Mail Code 138-78, California Institute of Technology, Pasadena, California 91125, USA
}

(Received 25 March 2005; published 1 August 2005)

\begin{abstract}
Nucleation is of great concern in many cases-for example, the production of artificial rainfall and the synthesis of advanced amorphous alloys. Although exact solutions have been well known to both homogeneous nucleation and heterogeneous nucleation occurring on a large flat container wall, yet in more general situations the actual nucleation takes place around finite-sized heterogeneous particles. The understanding of nucleation in such situations requires a more extended model which considers the size effect of nucleating agents. Partially motivated by our research on bulk metallic glasses, we construct such a geometric model. Also we derive an exact solution to the model and discuss briefly its physical implications. A previously presumed relation between the critical energy barrier $\left(E_{c}\right)$ and the volumetric Gibbs free energy of the critical nucleus $\left(G_{c}\right)$-i.e., $E_{c}=\frac{1}{2} G_{c}$-is found to be not true for general cases, although it is correct for the limiting cases.
\end{abstract}

DOI: 10.1103/PhysRevB.72.052101

Nucleation is a common phenomenon which plays a very important role in many research areas. The formation of rain droplets from clouds, the solidification of crystalline materials from their liquid states, and bone growth are all but a few isolated examples of nucleation phenomena. ${ }^{1}$ In some cases, such as the production of artificial rainfall, nucleation is beneficial and thus preferred, while in other cases-for example, the synthesis of organic ${ }^{2}$ or metallic glass ${ }^{3-5}$-nucleation is detrimental and has to be avoided. According to earlier studies by Volmer and Weber, ${ }^{6}$ Becker and Doring, ${ }^{7}$ and Turnbull and Fisher, ${ }^{8}$ for a small nucleus to grow from a parental phase, the size of the nucleus, $r$, and the energy associated with the formation of the nucleus, $E$, have to exceed a critical value $r_{c}$ and $E_{c}$, respectively. For homogeneous nucleation where there are no extrinsic nucleating agents, the classical solution to this critical problem is

$$
\begin{gathered}
r_{c}^{\mathrm{hom}}=\frac{2 \sigma}{G}, \\
E_{c}^{\mathrm{hom}}=\frac{16 \pi}{3} \frac{\sigma^{3}}{G^{2}},
\end{gathered}
$$

in which $\sigma$ is the interfacial energy per unit area (or the interfacial tension) between the new phase (i.e., the nucleus) and the parental phase, and $G$ is the Gibbs free energy difference per unit volume between the two phases. For heterogeneous nucleation which takes place on a large flat surface of an extrinsic object (such as a flat container wall or a flat substrate), the classical solution is

$$
\begin{gathered}
r_{c}^{h e t}=\frac{2 \sigma}{G}, \\
E_{c}^{h e t}=\frac{16 \pi}{3} \frac{\sigma^{3}}{G^{2}} f^{h e t}(\theta), \\
f^{h e t}(\theta)=\left[2-3 \cos \theta+\cos ^{3} \theta\right] / 4,
\end{gathered}
$$

where $\theta$ is the contact angle between the nucleus and the extrinsic object (for a quick review of these classical solutions, see Ref. 9). Nevertheless, in a large variety of cases, such as the formation of rain droplets from clouds or the nucleation of crystals from the interior of small-particle-
PACS number(s): 64.60.Qb, 81.05.Kf, 82.60.Nh, 05.70.Np

bearing liquids, ${ }^{10}$ the assumptions adopted in the derivation of the above two classical solutions (i.e., a superclean parental phase and/or a large flat extrinsic surface) are not well satisfied, and consequently these solutions cannot provide precise descriptions of such cases. The common feature of such cases is that there exist extrinsic nucleating agents whose sizes cannot be simply treated as either zero or infinity. ${ }^{11}$

In the field of metallic glass, for example, it has been shown that the glass forming ability (GFA) of an alloy upon cooling from its molten state is strongly influenced by the nucleating effect of the finite-sized impurities buried in the alloy melt. When the impurities are either fluxed ${ }^{12,13}$ or deactivated, ${ }^{14}$ the undercooling and GFA of the alloy can be improved dramatically. However, it is still not clear how exactly the impurity particles affect the nucleation process and how their effects can be carefully controlled by processing methods such as fluxing and microalloying. To help answer these questions, we present in this paper an extended geometric model for the critical problem of nucleation based on a finite-sized nucleating agent and then derive the exact solution and discuss its physical implications.

Figure 1(a) illustrates the geometric construction for the new model, where a nucleus $(N)$ forms at the interface between the parental phase $(P)$ and a finite-sized nucleating agent $(A) . O_{1}$ and $O_{2}$ are the spherical centers of $N$ and $A$, respectively. $S$ is a joint where the three phases $P, N$, and $A$ meet each other. Figure 1(b) is an illustration of the mechanical equilibrium at $S$, in which $\sigma, \sigma_{P A}$, and $\sigma_{N A}$ denote the interfacial tensions between $P$ and $N, P$ and $A$, and $N$ and $A$,

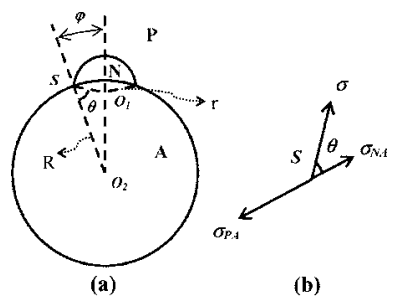

FIG. 1. (a) The geometric construction for the extended nucleation model, and (b) an illustration of the mechanical equilibrium at point $S$ in part (a). 
respectively. In both figures, $\theta$ is the contact angle between $N$ and $A$. Besides $\theta$, we introduce another important angle $\varphi$-i.e., $\angle \mathrm{SO}_{2} \mathrm{O}_{1}$ - to relate the radius of $\mathrm{A}\left(\mathrm{R}\right.$, i.e., $\mathrm{SO}_{2}$ ) with that of $N\left(r\right.$, i.e., $\left.S O_{1}\right)$.

With the above construction and denotations, it is trivial to obtain the following expressions.

The interfacial area (I.A.) between $P$ and $N$ [from Fig. 1(a)]:

$$
\text { I.A. } P N=2 \pi r^{2}[1-\cos (\varphi+\theta)] \text {. }
$$

The interfacial area between $N$ and $A$ [from Fig. 1(a)]:

$$
\text { I.A. }{ }_{N A}=2 \pi R^{2}(1-\cos \varphi) \text {. }
$$

The volume of $N$ [from Fig. 1(a)]:

$$
\begin{aligned}
V= & \frac{\pi}{3} r^{3}\left[2-3 \cos (\varphi+\theta)+\cos ^{3}(\varphi+\theta)\right] \\
& -\frac{\pi}{3} R^{3}\left(2-3 \cos \varphi+\cos ^{3} \varphi\right) .
\end{aligned}
$$

Interconnection among $r, R, \varphi$, and $\theta$ [from triangle $\mathrm{SO}_{1} \mathrm{O}_{2}$ in Fig. 1(a)]:

$$
\frac{r}{\sin \varphi}=\frac{R}{\sin (\varphi+\theta)} .
$$

Interconnection among $\sigma, \sigma_{P A}, \sigma_{N A}$, and $\theta$ [from Fig. 1(b)]:

$$
\sigma_{P A}-\sigma_{N A}=\sigma \cos \theta .
$$

Therefore, the energy change associated with the formation of nucleus $N$ is ( $G$ as defined earlier)

$$
\begin{aligned}
E & =\sigma \times \text { I.A } \cdot_{P N}+\left(\sigma_{N A}-\sigma_{P A}\right) \times \text { I.A } \cdot_{N A}-G \times V \\
& =\sigma \times \text { I.A } \cdot P N \\
& =E_{1}-E_{2},
\end{aligned}
$$

where

$$
E_{1}=2 \pi \sigma r^{2}[1-\cos (\varphi+\theta)]-2 \pi \sigma R^{2} \cos \theta(1-\cos \varphi)
$$

and

$$
\begin{aligned}
E_{2}= & \frac{\pi}{3} G r^{3}\left[2-3 \cos (\varphi+\theta)+\cos ^{3}(\varphi+\theta)\right] \\
& -\frac{\pi}{3} G R^{3}\left(2-3 \cos \varphi+\cos ^{3} \varphi\right) .
\end{aligned}
$$

For a given system, we have fixed $R$ and $\theta$. From Eq. (6) we get

$$
\frac{d r}{d \varphi}=\frac{R \sin \theta}{\sin ^{2}(\varphi+\theta)} .
$$

From Eqs. (9), (6), and (11), we get

$$
\begin{aligned}
\frac{d E_{1}}{d r} & =2 \pi \sigma R^{2} \frac{d}{d r}\left\{\frac{\sin ^{2} \varphi}{\sin ^{2}(\varphi+\theta)}[1-\cos (\varphi+\theta)]-\cos \theta(1-\cos \varphi)\right\} \\
& =2 \pi \sigma R^{2} \frac{d \varphi}{d r} \frac{d}{d \varphi}\left[\frac{\sin ^{2} \varphi}{1+\cos (\varphi+\theta)}-\cos \theta(1-\cos \varphi)\right] \\
& =2 \pi \sigma R \frac{\sin ^{2}(\varphi+\theta)}{\sin \theta} \sin \varphi\left\{\frac{2 \cos \varphi}{1+\cos (\varphi+\theta)}+\frac{\sin \varphi \sin (\varphi+\theta)}{[1+\cos (\varphi+\theta)]^{2}}-\cos \theta\right\} \\
& =2 \pi \sigma r \sin (\varphi+\theta) \frac{1-\cos ^{2}(\varphi+\theta)}{\sin \theta}\left\{\frac{2 \cos \varphi}{1+\cos (\varphi+\theta)}+\frac{\sin \varphi \sin (\varphi+\theta)}{[1+\cos (\varphi+\theta)]^{2}}-\cos \theta\right\} \\
& =2 \pi \sigma r \frac{1-\cos (\varphi+\theta)}{\sin \theta}\{2 \cos \varphi \sin (\varphi+\theta)+\sin \varphi[1-\cos (\varphi+\theta)]-\cos \theta \sin (\varphi+\theta)[1+\cos (\varphi+\theta)]\} \\
& =2 \pi \sigma r \frac{1-\cos (\varphi+\theta)}{\sin \theta}\{2[\sin \theta+\sin \varphi \cos (\varphi+\theta)]+\sin \varphi[1-\cos (\varphi+\theta)]-\cos \theta \sin (\varphi+\theta)[1+\cos (\varphi+\theta)]\} \\
& =2 \pi \sigma r \frac{1-\cos (\varphi+\theta)}{\sin \theta}\{2 \sin \theta+\sin \varphi[1+\cos (\varphi+\theta)]-\cos \theta \sin (\varphi+\theta)[1+\cos (\varphi+\theta)]\} \\
& =2 \pi \sigma r \frac{1-\cos (\varphi+\theta)}{\sin \theta}\{2 \sin \theta-\sin \theta \cos (\varphi+\theta)[1+\cos (\varphi+\theta)]\} \\
& =2 \pi \sigma r[2-3 \cos (\varphi+\theta)+\cos 3(\varphi+\theta)] .
\end{aligned}
$$

From Eqs. (10) and (6) we get 


$$
\begin{aligned}
\frac{d E_{2}}{d r}=\frac{\partial E_{2}}{\partial r}+\frac{\partial E_{2}}{\partial \varphi} \frac{d \varphi}{d r}= & \pi G r^{2}\left[2-3 \cos (\varphi+\theta)+\cos ^{3}(\varphi+\theta)\right]+\frac{d \varphi}{d r}\left\{\frac{\pi}{3} G r^{3}[3 \sin (\varphi+\theta)\right. \\
& \left.\left.-3 \cos ^{2}(\varphi+\theta) \sin (\varphi+\theta)\right]-\frac{\pi}{3} G R^{3}\left(3 \sin \varphi-3 \cos ^{2} \varphi \sin \varphi\right)\right\} \\
= & \pi G r^{2}\left[2-3 \cos (\varphi+\theta)+\cos ^{3}(\varphi+\theta)\right]+\pi G \frac{d \varphi}{d r}\left[r^{3} \sin ^{3}(\varphi+\theta)-R^{3} \sin ^{3} \varphi\right] \\
= & \pi G r^{2}\left[2-3 \cos (\varphi+\theta)+\cos ^{3}(\varphi+\theta)\right] .
\end{aligned}
$$

Therefore, from Eqs. (8), (12), and (13), we have

$$
\begin{aligned}
\frac{d E}{d r}=\frac{d E_{1}}{d r}-\frac{d E_{2}}{d r} & =2 \pi \sigma r\left[2-3 \cos (\varphi+\theta)+\cos ^{3}(\varphi+\theta)\right]-\pi G r^{2}\left[2-3 \cos (\varphi+\theta)+\cos ^{3}(\varphi+\theta)\right] \\
& =\pi r(2 \sigma-G r)\left[2-3 \cos (\varphi+\theta)+\cos ^{3}(\varphi+\theta)\right] .
\end{aligned}
$$

The critical condition is $d E /\left.d r\right|_{r_{c}}=0$; hence, we get

$$
r_{c}=\frac{2 \sigma}{G}
$$

It is clear that the critical diameter of the nucleus does not depend on either the contact angle $\theta$ or the nucleating-agent size $R$ and, that it has the same value for the present extended case and for the two classical cases-i.e., $r_{c}=r_{c}^{\text {hom }}=r_{c}^{\text {het }}$.

To find out the critical energy barrier $E_{c}$, we substitute $r_{c}$ into Eqs. (9), (10), and then (8). We get

$$
\begin{gathered}
\left.E_{1}\right|_{r_{c}}=8 \pi \frac{\sigma^{3}}{G^{2}}\left[1-\cos (\varphi+\theta)-\frac{\sin ^{2}(\varphi+\theta)}{\sin ^{2} \varphi} \cos \theta(1-\cos \varphi)\right], \\
\left.E_{2}\right|_{r_{c}}=\frac{8 \pi}{3} \frac{\sigma^{3}}{G^{2}}\left[2-3 \cos (\varphi+\theta)+\cos ^{3}(\varphi+\theta)-\frac{\sin ^{3}(\varphi+\theta)}{\sin ^{3} \varphi}\left(2-3 \cos \varphi+\cos ^{3} \varphi\right)\right],
\end{gathered}
$$

and

$$
E_{c}=\left.E_{1}\right|_{r_{c}}-\left.E_{2}\right|_{r_{c}}=\frac{16 \pi}{3} \frac{\sigma^{3}}{G^{2}} g(R, \theta),
$$

where

$$
\begin{aligned}
g(R, \theta)= & \frac{1}{2}\left[1-\cos ^{3}(\varphi+\theta)-3 \cos \theta \frac{\sin ^{2}(\varphi+\theta)}{1+\cos \varphi}\right. \\
& \left.+\frac{\sin ^{3}(\varphi+\theta)}{\sin ^{3} \varphi}\left(2-3 \cos \varphi+\cos ^{3} \varphi\right)\right]
\end{aligned}
$$

and $\varphi=\varphi(R, \theta)$ is determined by Eq. (6) or, explicitly,

$$
\varphi=\operatorname{arccot}\left(\frac{R / r_{c}-\cos \theta}{\sin \theta}\right) .
$$

Figures 2(a), 2(b), and 2(c) present the three-dimensional (3D) image and some $2 \mathrm{D}$ projected curves of this bivariate function $g(R, \theta)$ with $R$ scaled by $r_{c}=2 \sigma / G$. From these figures, as well as from Eqs. (19) and (20), it can be seen that for any fixed contact angle $\theta$ (i.e., fixed type of nucleating agent), as the agent size $R$ goes to $0,{ }^{15} g(R, \theta)$ goes to 1 , corresponding to $E_{c}$ going to $E_{c}^{\mathrm{hom}}=(16 \pi / 3) \sigma^{3} / G^{2}$, which means nucleation occurs in a homogeneous manner in the limiting case where $R=0$. Also, for any fixed $\theta$, as $R$ goes to $+\infty, g(R, \theta)$ goes to a constant value $g(+\infty, \theta)$. It is trivial to find the expression for $g(+\infty, \theta)$ through Eqs. (19) and (20) (Ref. 15):

$$
\begin{aligned}
g(+\infty, \theta) & =\frac{1}{2}\left(1-\cos ^{3} \theta-\frac{3}{2} \cos \theta \sin ^{2} \theta\right) \\
& =\frac{1}{4}\left(2-3 \cos \theta+\cos ^{3} \theta\right) \\
& =f^{\text {het }}(\theta) .
\end{aligned}
$$

Therefore, the classical heterogeneous solution-i.e., Eq. (2) - actually describes only the limiting case of extended model, where the agent size tends to infinity. Although this limiting solution may be at the same time a good estimate for $g(R, \theta)$ when $R$ is significantly larger than the critical nucleus diameter $r_{c}$ [by at least two or three orders of magnitude according to Fig. 2(b)], it cannot be used to depict a large category of nucleation processes occurring at low "undercoolings" of a parental phase. ${ }^{16}$ This is because at low undercoolings, the Gibbs free-energy difference between the parental phase and the new phase is very small and thus the critical nucleus diameter is very large according to $r_{c}$ $=2 \sigma / G$, as a result of which a finite nucleating agent cannot be readily considered significantly larger than $r_{c}$. Therefore, in such general cases, the present model should be considered.

Besides the nucleating-agent size effect, this extended model also releases new information about the dependence of the critical energy barrier on the contact angle $\theta$. The classical heterogeneous solution-i.e., Eq. (2)—predicts that the critical energy barrier drops from $E_{c}^{\text {hom }}$ to 0 as $\theta$ de- 


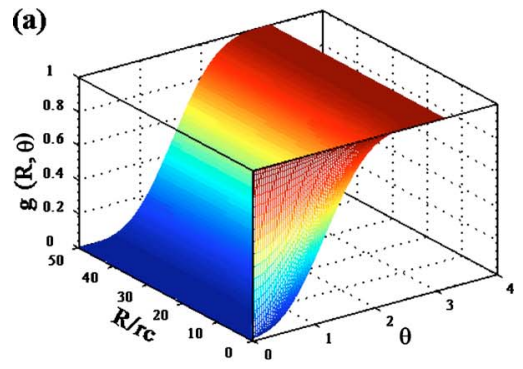

(b)

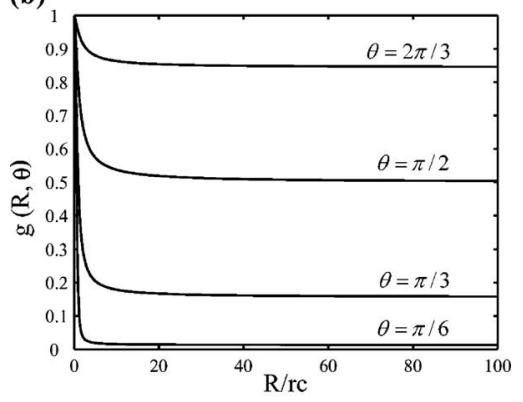

(c)



FIG. 2. (Color online) (a) 3D image of the bivariate function $g(R, \theta)$, (b) 2D plots of $g(R, \theta)$ vs $R / r_{c}$ at different values of $\theta$, and (c) 2D plots of $g(R, \theta)$ vs $\theta$ at different values of $R / r_{c}$.

creases from $\pi$ to 0 . However, in the present extended model, the conclusion is somewhat different. For conve- nience sake, here we consider another form of Eq. (19):

$$
\begin{aligned}
g(R, \theta)= & \frac{1}{2}\left[1-\cos ^{3}(\varphi+\theta)-3\left(\frac{R}{r_{c}}\right)^{2} \cos \theta(1-\cos \varphi)\right. \\
& \left.+\left(\frac{R}{r_{c}}\right)^{3}\left(2-3 \cos \varphi+\cos ^{3} \varphi\right)\right],
\end{aligned}
$$

since we fix the value of $R$ in order to study the contribution of varying $\theta$. As can been seen from Fig. 2(c), as well as from Eqs. (20) and (22), ${ }^{17}$ for any fixed $R(0 \leqslant R<\infty)$, as $\theta$ tends to $\pi, g(R, \theta)$ always tends to 1 and thus nucleation always tends to occur in a homogeneous manner. Nevertheless, as $\theta$ tends to 0 , the value of $g(R, \theta)$ depends on whether $R \geqslant r_{c}$ or $R<r_{c}$. If $R \geqslant r_{c}, g(R, \theta)$ always tends to 0 , meaning the nucleation energy barrier disappears at $\theta=0$; if $R<r_{c}$, $g(R, \theta)$ tends to a finite value $g(R, 0)=1-3\left(R / r_{c}\right)^{2}$ $+2\left(R / r_{c}\right)^{3}$ as determined by Eqs. (20) and (22). In the latter case, the smaller the ratio $R / r_{c}$, the closer $g(R, 0)$ is to 1 -i.e., the closer the nucleation process is to the homogeneous case, even though $\theta=0$.

It is also noteworthy that the previously presumed relation between the critical energy barrier $\left(E_{c}\right)$ and the volumetric Gibbs free energy of the critical nucleus $\left(\left.E_{2}\right|_{r_{c}}\right): E_{c}=\left.\frac{1}{2} E_{2}\right|_{r_{c}}$ does not necessarily hold for a finite $R$ in the present extended model [see Eqs. (17)-(19)], although it is correct in the two limiting cases-i.e., when $R=0$ or $R=+\infty$.

In sum, we have presented an extended geometric model for the critical problem of nucleation by introducing the size effect of an extrinsic nucleating agent. The classical solutions to homogeneous and heterogeneous critical problems have been proven to be limiting cases of this extended model. Since in many cases the limiting conditions adopted in the derivations of the two classical solutions are not satisfied, this present model is expected to provide a more complete and more reliable description for general nucleation phenomena.
*Corresponding author. Electronic address: xudh@ caltech.edu

${ }^{1}$ A. G. Walton, Science 148, 601 (1965).

${ }^{2}$ C. A. Angell, Science 267, 1924 (1995).

${ }^{3}$ A. L. Greer, Science 267, 1947 (1995).

${ }^{4}$ F. Spaepen et al., Annu. Rev. Phys. Chem. 35, 241 (1984).

${ }^{5}$ D. Turnbull, Contemp. Phys. 10, 473 (1969).

${ }^{6} \mathrm{M}$. Volmer et al., Z. Phys. Chem., Stoechiom. Verwandtschaftsl. 119, 277 (1926).

${ }^{7}$ R. Becker and W. Doring, Ann. Phys. (Leipzig) 24, 719 (1935).

${ }^{8}$ D. Turnbull and J. C. Fisher, J. Chem. Phys. 17, 71 (1949).

${ }^{9}$ D. A. Porter and K. E. Easterling, Phase Transformations in Metals and Alloys, 2nd ed. (Chapman \& Hall, London, 1992).

${ }^{10}$ Whether given particles should be considered large or small depends on the nucleation temperature or the undercooling of the liquids; the current small-particle situation is easily encountered at high temperatures-i.e., low undercoolings-which is generally true for nonglassy systems.

${ }^{11}$ Homogeneous nucleation can be considered a case where the size of the extrinsic nucleating agent is zero; classical heterogeneous nucleation can be considered a case where the size of the nucleating agent is infinity. This will be elaborated in the following context.

${ }^{12}$ H. W. Kui et al., Appl. Phys. Lett. 45, 615 (1984).

${ }^{13}$ D. M. Herlach et al., J. Non-Cryst. Solids 250, 271 (1999).

${ }^{14}$ Z. P. Lu et al., Appl. Phys. Lett. 83, 2581 (2003).

${ }^{15}$ According to Eq. (20), for a fixed $\theta(0 \leqslant \theta \leqslant \pi), R$ going to 0 is equivalent to $\varphi$ going to $\pi-\theta$, and $R$ going to $+\infty$ is equivalent to $\varphi$ going to 0 .

${ }^{16}$ Here, "undercooling" refers to the deviation from the thermodynamic equilibrium between the parental phase and the new phase such that the following discussion not only applies to the crystallization-of-liquid case but also to most other cases.

${ }^{17}$ According to Eq. (20), for any fixed $R(0 \leqslant R<\infty), \theta$ going to $\pi$ is equivalent to $\varphi$ going to 0 . For a fixed $R\left(R>r_{c}\right), \theta$ going to 0 is equivalent to $\varphi$ going to 0 . For a fixed $R\left(R<r_{c}\right), \theta$ going to 0 is equivalent to $\varphi$ going to $\pi$. If $R=r_{c}, \theta$ going to 0 is equivalent to $\varphi$ going to $\pi / 2$. 